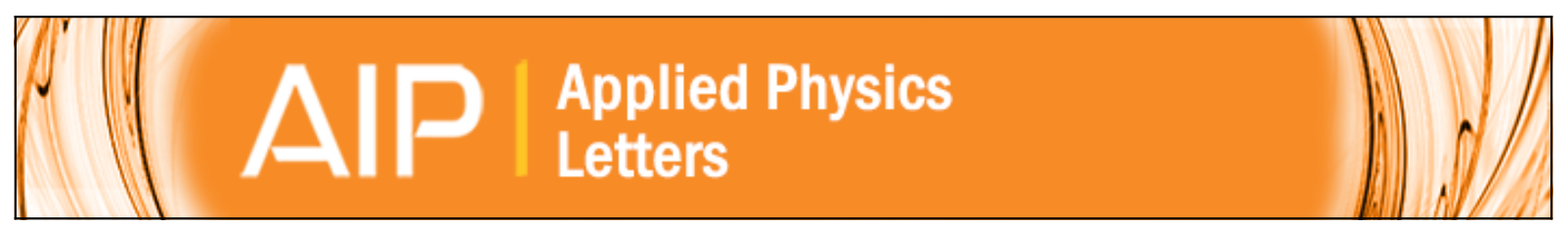

\title{
Nonlinear dissipation in diamond nanoelectromechanical resonators
}

Matthias Imboden, Oliver Williams, and Pritiraj Mohanty

Citation: Applied Physics Letters 102, 103502 (2013); doi: 10.1063/1.4794907

View online: http://dx.doi.org/10.1063/1.4794907

View Table of Contents: http://scitation.aip.org/content/aip/journal/apl/102/10?ver=pdfcov

Published by the AIP Publishing

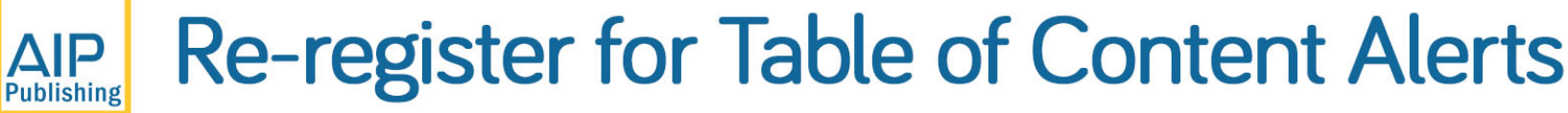

Create a profile.

Sign up today! 


\title{
Nonlinear dissipation in diamond nanoelectromechanical resonators
}

\author{
Matthias Imboden, ${ }^{1}$ Oliver Williams ${ }^{2}$ and Pritiraj Mohanty ${ }^{1, a)}$ \\ ${ }^{1}$ Department of Physics, Boston University, 590 Commonwealth, Boston, Massachusetts 02215, USA \\ ${ }^{2}$ School of Physics and Astronomy, Cardiff University, Queen's Buildings, The Parade, Cardiff CF24 3AA, \\ United Kingdom
}

(Received 1 January 2013; accepted 25 February 2013; published online 11 March 2013)

\begin{abstract}
Dissipation of energy in micro- and nano-electromechanical resonators governs their dynamical response and limits their potential use in device applications. Quantified by the quality factor $Q$, dissipation $\left(Q^{-1}\right)$ usually occurs by energy loss mechanisms that are linear, appearing as a damping term proportional to the velocity. Mechanisms of linear dissipation in micro- and nano-mechanical resonators are well studied both theoretically and experimentally. Mechanisms of nonlinear dissipation of energy, however, are rarely studied, though their effects could be fundamentally important to the operation of numerous devices based on nonlinear resonators such as switches, signal processers, sensors, and energy harvesting systems. Here, we report experimental observation of nonlinear dissipation in diamond nanoelectromechanical resonators. (C) 2013 American Institute of Physics. [http://dx.doi.org/10.1063/1.4794907]
\end{abstract}

Microelectromechanical systems (MEMS) and nanoelectromechanical systems (NEMS) are often based on resonators of corresponding length scales for many device applications that are of fundamental and technical interests. MEMS and NEMS resonators are used for signal processing in wireless communications in filters, timing oscillators and switches, and as sensors in accelerometers and gyroscopes in inertial navigation. Fundamental applications of NEMS resonators include the effort to observe quantum effects in macroscopic systems and spintronics. Even though resonators are used in the linear regime in all these applications, resonators operating in the nonlinear regime are also used in numerous counterintuitive applications that include signal processing using stochastic resonance, ${ }^{1}$ improved sensitivity, ${ }^{2}$ and energy harvesting. ${ }^{3}$ The understanding of nonlinear dissipation mechanisms is therefore fundamentally important for determining the limitations of devices using resonators in either linear or nonlinear regimes.

Dissipation mechanisms are usually expressed by the following nonlinear damped driven resonator equation. The damping term is proportional to the velocity of the resonator, where a phenomenological parameter $\gamma$ represents damping given by a particular mechanism. This term characterizes the normalized rate at which energy escapes a particular resonant mode. All underlying physical mechanisms are bundled into this single phenomenological parameter. The simplest way to add mechanical nonlinearity is given by the Duffing equation, which includes a higher order term in $x$

$$
\ddot{x}+\gamma \dot{x}+\frac{k}{m} x+\frac{k_{3}}{m} x^{3}=\frac{F}{m} \cos (\omega t)
$$

where $k_{3}$ is the nonlinear coefficient. An $x^{2}$ term may be included but the $x^{3}$ term is required to ensure the energy is bounded. A positive $k_{3}$ makes the structure stiffer and results in maximum amplitudes at higher frequencies. Correspondingly, a negative $k_{3}$ results in beam softening and lowers the frequency

${ }^{a)}$ E-mail: mohanty@bu.edu. of the maximum displacement; these cases are described by the backbone curve. After a critical drive force, the resonator displacement bifurcates, resulting in a parameter space where the total displacement depends on the history of the resonator. This can be observed by changing the sweep direction of the drive frequency. This behavior has led to the study of controlled switching between stable states, ${ }^{4,5}$ stochastic resonance, ${ }^{6-8}$ and control of nonlinearity in resonator devices. ${ }^{9}$

Intrinsic nonlinearities of the resonator material typically contribute only at displacements far greater than nonlinear effects due to the geometry or external potentials. An example of nonlinearities due to an external potential can be illustrated by electrostatic actuation and detection method. For weak drive forces,

$$
\ddot{x}+\gamma \dot{x}+\left(\omega_{0}^{2}-\frac{C^{\prime \prime} V_{B}^{2}}{2 m}\right) x \approx \frac{C^{\prime} V_{B} V_{d}}{m} .
$$

The drive force results from a Taylor expansion of the voltage acting between the resonator and the gates that form the capacitor. Simply including higher order terms in $x$ will result in terms proportional to $x^{2}$ and $x^{3}$, resulting in an explicit term for $k_{3}$ (and $k_{2}$, where applicable). Geometric nonlinearities occur when the thin-beam approximation is no longer valid. ${ }^{10}$ For a doubly clamped rectangular beam, it can be shown that the nonlinearity coefficient takes the form $k_{3}=\omega_{n}^{2} \frac{S}{2 I} \beta_{n}$, where $\omega_{n}$ is the n-th mode eigenfrequency, $S$ is the cross sectional area of the beam, and $I$ the second moment of inertia. $\beta_{n}$ is a mode dependent numerical constant, where $\beta_{1} \approx 0.2$ and approaches $1 / 2$ as $n \rightarrow \infty$. Typical displacements, $<10 \mathrm{~nm}$, are small compared to device widths or thicknesses that are usually greater than $100 \mathrm{~nm}$. So, with the exception of graphene and CNT devices, nonlinearities due to external potentials will often dominate over geometric nonlinearities.

One may consider the additional terms $\dot{x}^{3}, \dot{x} x^{2}, x^{2} \dot{x}$, which are all on the same order as $x^{3}$. As these terms are made up of the velocity, they result in nonlinear dissipative contributions. The following is known as the van der Pol-Duffing equation: 


$$
m \ddot{x}+m \gamma \dot{x}+k x+k_{3} x^{3}+\eta x^{2} \dot{x}=F \cos (\omega t),
$$

where $\eta$ is the nonlinear damping coefficient and $F$ the drive force at frequency $\omega$. An approximate solution ${ }^{10}$ can be obtained using secular perturbation theory, valid in the limit of low dissipation and small oscillations. In addition, it is assumed that the response is only large close to the resonance frequency and hence the perturbation is conducted close to the resonance. The nonlinear oscillator displacement amplitude becomes

$$
x_{0}^{2}=\frac{\left(\frac{F}{2 m \omega_{0}^{2}}\right)^{2}}{\left(\frac{\omega-\omega_{0}}{\omega_{0}}-\frac{3}{8} \frac{k_{3}}{m \omega_{0}^{2}} x_{0}^{2}\right)^{2}+\left(\frac{1}{2} Q^{-1}+\frac{1}{8} \frac{\eta}{m \omega_{0}} x_{0}^{2}\right)^{2}} .
$$

In contrast to the linear response, the maximum displacement is now shifted from the resonance frequency, where the frequency shift is dependent on $x^{2}$. This results in the backbone expression given by $\omega_{\max }=\omega_{0}+3 k_{3}\left(x_{0}\right)_{\max }^{2} / 8 m \omega_{0}$. For increasing drive force, Eq. (4) eventually bifurcates resulting in a frequency space that is multivalued in $x_{0}$. The critical amplitude (and corresponding force and frequency) is defined by the onset of the bifurcation ${ }^{10}$

$$
x_{c 0}^{2}=\frac{8}{3} \frac{m \gamma \omega_{0}}{k_{3}} \frac{1}{\sqrt{3}-\frac{\eta \omega_{0}}{k_{3}}} .
$$

For $\eta=0, x_{c 0}^{2}$ is inversely proportional to $k_{3}$. As $k_{3}$ approaches zero (which represents the linear regime), the amplitude diverges before bifurcation occurs. This is the expected result. As the nonlinear damping increases, the critical displacement also increases and even diverges as $\eta \rightarrow \sqrt{3} k_{3} / \omega_{0}$ above which no bifurcation in frequency space occurs. This is a fundamental difference compared to the case without nonlinear damping, where bifurcation will eventually always occur for nonzero values of $k_{3}$.

To demonstrate the effects of nonlinear damping, our experiments were conducted on a set of resonators designed to operate with the electrostatic or magnetomotive method. The two sets of resonators shown in Figure 1 were fabricated using e-beam lithography from nano- and ultranano-crystalline diamond (NCD and UNCD). The doubly clamped NCD resonator shown in Figure 1(a) is sandwiched between two side gates for capacitive actuation as schematically shown in Figure 1(c). This resonator is $380 \mathrm{~nm}$ thick, $500 \mathrm{~nm}$ wide and $18.6 \mu \mathrm{m}$ long. The resulting fundamental flexural mode has a frequency of $20.917 \mathrm{MHz}$ and linear quality factor at room temperature of 6845 (without circuit loading). The Young's modulus of $\sim 600 \mathrm{GPa}$ is low for diamond due to the large undercut at the clamps, resulting in an effectively longer structure. The true Young's modulus of the diamond has been independently determined ${ }^{11}$ to be as high as $1100 \mathrm{GPa}$. Figure 1(b) depicts a series of doubly clamped UNCD beams with gold top electrodes for magnetomotive actuation, as schematically shown in Figure 1(d). The resonator discussed here is $340 \mathrm{~nm}$ thick, $350 \mathrm{~nm}$ wide, and $13.1 \mu \mathrm{m}$ long, with a resonant frequency of $13.501 \mathrm{MHz}$ and a quality factor at $40 \mathrm{mK}$ of $\sim 14920$ (without circuit loading). ${ }^{12}$ The resonators were cooled to $40 \mathrm{mK}$ in a dilution cryostat and actuated magnetomotively with a superconducting magnet. A full description of these structures and their linear dynamic response is given elsewhere. $^{13}$

Experimentally, there are multiple ways the effect of nonlinear damping may be observed. For low drive forces, as compared to the critical force, the response is essentially Lorentzian, one may observe a widening of the resonance given by $\Delta \omega=\gamma+\omega_{0} \eta x_{0}^{2} / 4$, and correspondingly a
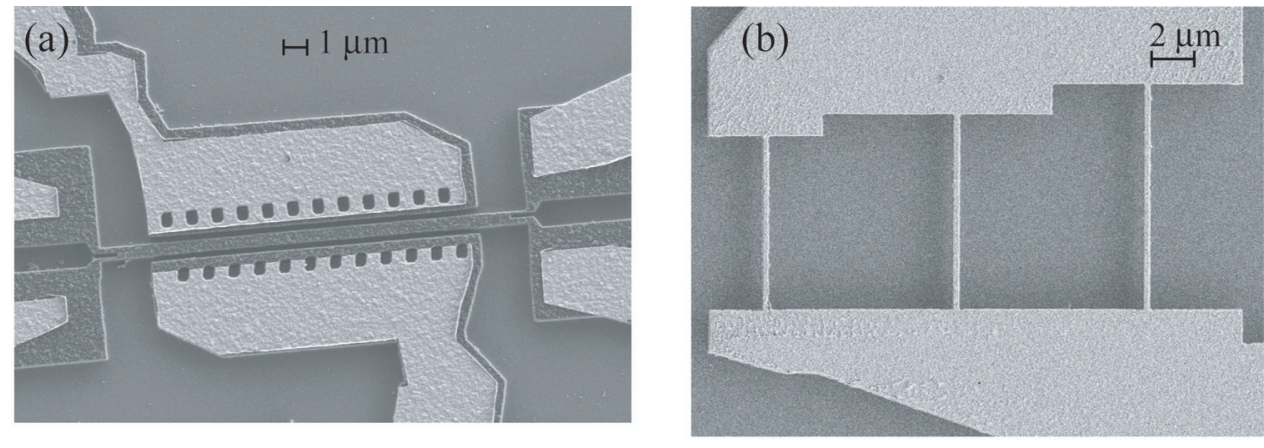

(c)

(d)

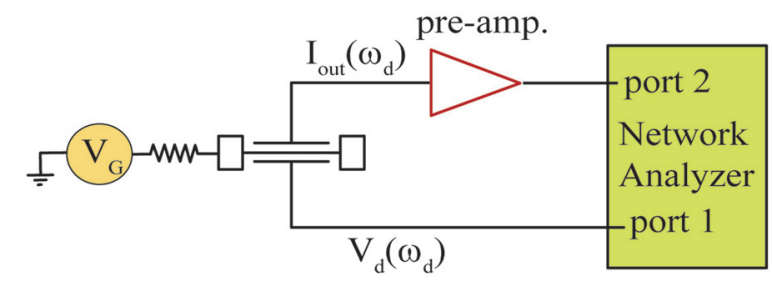

Network Analyzer

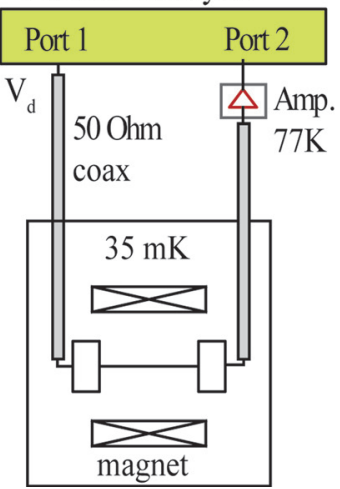

FIG. 1. (a) NCD doubly clamped beam with capacitive side electrodes. (b) UNCD harp structure with multiple doubly clamped beams of varying lengths, similar to the one discussed here. (c) Capacitive drive and detection schematics. (d) Magnetomotive drive and detection schematics. For all measurements, the resonators are actuated in a high vacuum were viscous damping does not occur. 
displacement dependent dissipation of the form $Q^{-1} \cong \frac{\gamma}{\omega_{0}}$ $+\eta x_{0}^{2} / 4$. Typically, this is a small effect that is hard to observe in experiments as the uncertainty in $\Delta \omega$ can be significant. However, the effect of $\eta$ can be also observed in the high drive regime where the response bifurcates and the correction to the amplitude is significant. ${ }^{10}$ It is shown that for $\eta=0$ the normalized response (where the amplitude is divided by the drive force) is independent of the drive force. For $\eta>0$, however, the normalized response drops with increasing drive force, as depicted in Figure 2(b), implying that the maximum amplitude response is not affected by $k_{3}$ and Hooke's law still applies. Correspondingly, if nonlinear damping is present a displacement-force trace will no longer fall on a straight line but fall off at higher drive forces. In both cases, bifurcation occurs above a critical drive force as the nonlinear dissipation is not sufficient to suppress this phenomenology $\left(\eta<\sqrt{3} k_{3} / \omega_{0}\right)$.

Figure 3 depicts a power sweep of an electrostatically driven diamond resonator at room temperature (a) and a magnetomotively driven doubly clamped diamond resonator at $40 \mathrm{mK}$ (b). Figure 3(a) shows the normalized power sweep of an in-plane flexural mode in the doubly clamped structure
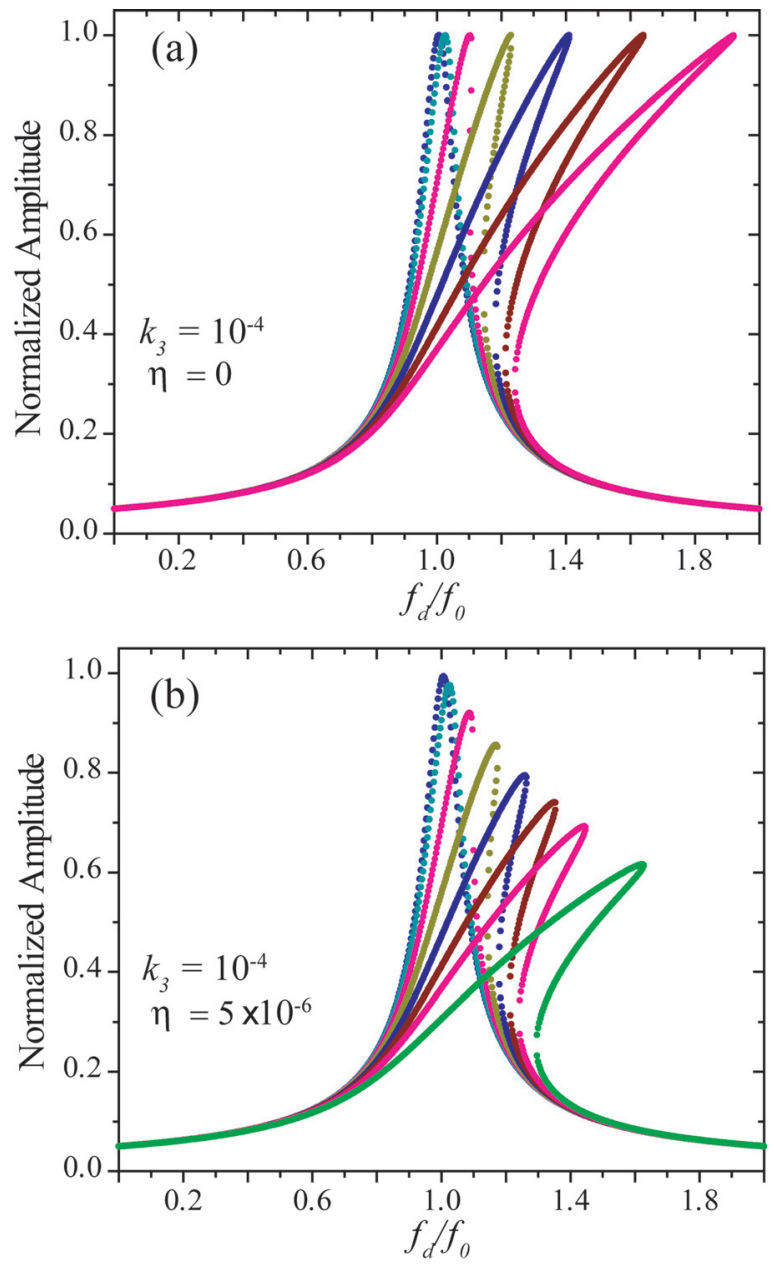

FIG. 2. (a) Normalized response of the Duffing equation without nonlinear damping. This is the numerical solution the force-displacement relation given by Eq. (4), and no nonlinear dissipation $(\eta=0)$. For forces above the critical force, the solution becomes triple valued, where middle amplitude solution is not physical. Colors indicate varying drive forces. (b) Same as above but now including finite nonlinear damping as described by the Pol-Duffing equation.
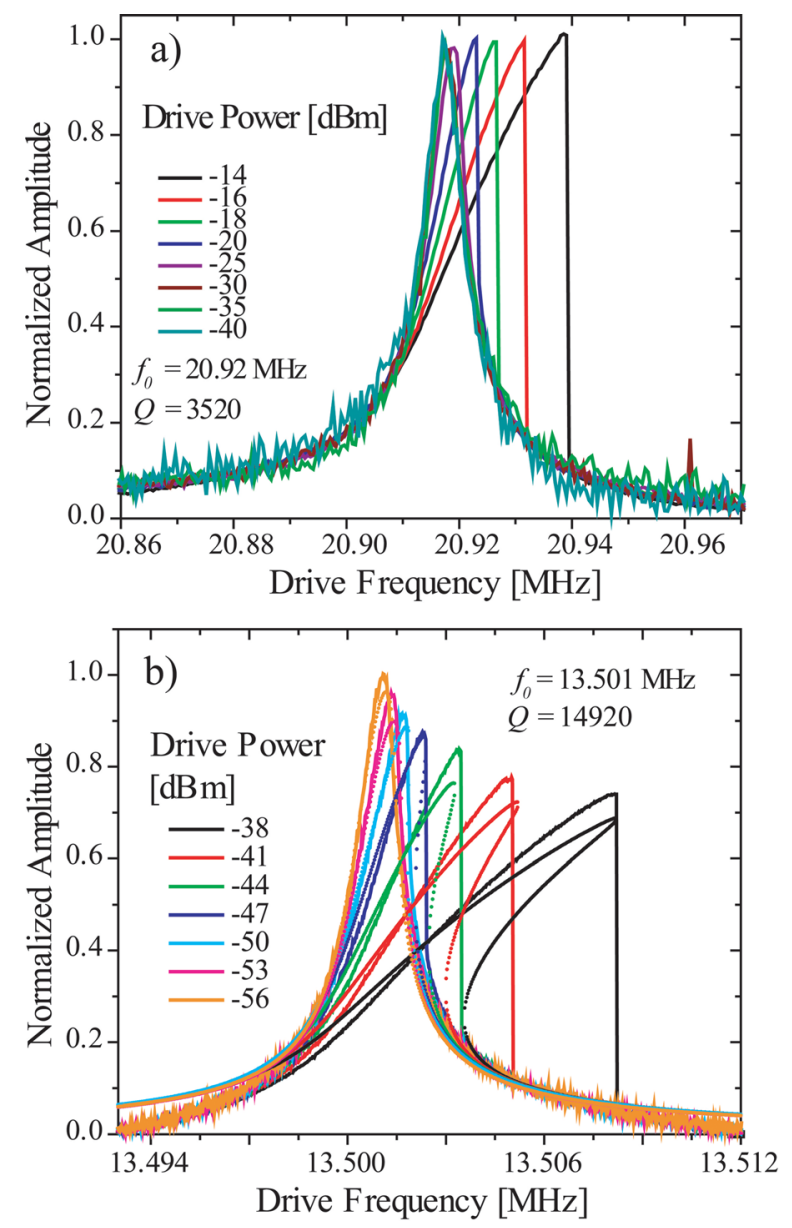

FIG. 3. (a) Normalized amplitude response of a doubly clamped structure driven electrostatically at room temperature. $f_{0}=21.05 \mathrm{MHz}, Q=3720$, $\eta=0$, in-plane flexural mode. In this case, $\eta=0$ and the normalized maximum amplitude is constant as seen in Fig. 2(a). (b) Normalized drive power sweep of doubly clamped structure driven magnetomotively in a dilution cryostat at $40 \mathrm{mK} . f_{0}=13.5 \mathrm{MHz}, Q=14924, k_{3}=4.5$ (normalized units). Here, $\eta=4.8 \times 10^{-6}$, for this out-of-plane flexural mode and a clear drop in the maximum amplitude is observed.

driven electrostatically with $f_{0}=20.92 \mathrm{MHz}, Q=3520$, $\eta=0$. Figure 3(b) shows normalized power sweeps of the out-of-plane flexural mode in the doubly clamped structure driven magnetomotively, with $f_{0}=13.5 \mathrm{MHz}, Q=14924$, $k_{3}=4.5$, and $\eta=4.8 \times 10^{-6}$ (normalized units). Again, bifurcation was observed in both cases, indicating that the nonlinear dissipation is still in the weak limit.

Ideally, all traces should be reproduced by Eq. (4), provided the actuation force is known. In reality, the fits proved very difficult and results were only obtained by allowing for different values for $k_{3}$ and $\eta$ for each trace. Hence, the solid lines in Figure 3 should only be considered as a guide to the eye rather than exact fits. These results show that the effects of nonlinear damping correlate well with the theory presented in Figure 2. Why more precise fitting was not possible may indicate that further mechanisms that are not accounted for are at play. For comparison, the trace in Figure 3(a) shows results for the electrostatically driven beam at room temperature. Even though the response is highly nonlinear, there is no measurable nonlinear damping. The normalized maximum amplitude is constant, and no peak broadening is observed within experimental errors. 
For large drive forcing, it is possible to raise the temperature of the resonator through Ohmic heating. Corresponding the quality factor would decrease. In our experiment, we can rule out this heating effect and the source of increased dissipation with drive power. For the dissipation to increase by a factor of 1.4, the observed temperature would have to rise from $40 \mathrm{mK}$ to roughly $500 \mathrm{mK}$. At $500 \mathrm{mK}$, the expected frequency shift would exceed $6 \mathrm{kHz}$, which is not observed here.

$Q$ is much higher at low temperatures, which results in larger amplitudes for the equivalent drive force. The drive powers cannot be compared directly as the transduction efficiency of magnetomotive and electrostatic methods are very different. In principle, only the absolute amplitudes should factor in.

Similar nonlinear effects have been observed in NEMS devices. It is conjectured that nonlinearities due to geometry may result in nonlinear dissipation. ${ }^{14}$ However, such mechanisms are still unknown. While smaller resonators are often easier to drive into the nonlinear regime, larger structures also have shown frequency broadening as a function of increased strain. ${ }^{15}$ These effects are considerable, but their precise cause is still unknown. It is found ${ }^{16}$ that parametric amplification can be limited by nonlinear damping as was observed in a recent experiment on qubit-coupled nanoresonator. In addition, nonlinear dissipation must be considered in active feedback systems, where out of phase feedback will result in nonlinear dissipation ${ }^{17}$ as well as noise squeezing. ${ }^{18}$

Strong nonlinear damping has recently been observed in CNT and graphene resonators under tensile strain and string like structures. ${ }^{19}$ In these structures, nonlinear damping is responsible for destroying hysteresis. The frequency widening is greater than the frequency shift and is analyzed in the zero linear dissipation limit, where $\Delta f \propto\left(\eta^{1 / 3} / m\right)\left(F_{\text {drive }} / f_{0}\right)^{2 / 3}$. While again, no particular damping mechanism is discovered, it is suggested that linear damping mechanisms coupled to geometric nonlinearities or external mechanisms such as clamping losses may contribute to the effect. The relatively high nonlinear damping may also point to a mechanism specific to graphene and CNT such as sliding of the carbon resonator over its metal electrodes. In experiments ${ }^{20}$ involving two-level systems (TLS) the observed strain dependent dissipation and shifts in sound velocity can be explained by coupling of TLS. This mechanism could be a good candidate as a source of nonlinear dissipation as TLS are known to be significant in both the diamond structure presented above and are likely also to occur in the graphene and CNT resonators that have exhibited nonlinear dissipation.

OAW is funded by the Marie Curie fellowship "DIAMEMS" and ESPRC grant "NCD_MEMS".

${ }^{1}$ D. Guerra, A. Bulsara, W. Ditto, S. Sinha, K. Murali, and P. Mohanty, Nano Lett. 10, 1168 (2010).

${ }^{2}$ J. Aldridge and A. Cleland, Phys. Rev. Lett. 94, 156403 (2005).

${ }^{3}$ F. Cottone, H. Vocca, and L. Gammaitoni, Phys. Rev. Lett. 102, 80601 (2009).

${ }^{4}$ R. Badzey, G. Zolfagharkhani, A. Gaidarzhy, and P. Mohanty, Appl. Phys. Lett. 85, 3587 (2004).

${ }^{5}$ D. Guerra, M. Imboden, and P. Mohanty, Appl. Phys. Lett. 93, 033515 (2008).

${ }^{6}$ R. L. Badzey and P. Mohanty, Nature 437, 995 (2005).

${ }^{7}$ D. Guerra, T. Dunn, and P. Mohanty, Nano Lett. 9, 3096 (2009)

${ }^{8}$ T. Dunn, D. Guerra, and P. Mohanty, Eur. Phys. J. B 69, 5 (2009).

${ }^{9}$ R. B. Karabalin, M. C. Cross, and M. L. Roukes, Phys. Rev. B 79, 165309 (2009).

${ }^{10} \mathrm{R}$. Lifshitz and M. Cross, Reviews of Nonlinear Dynamics and Complexity (Wiley-VCH, 2008), Vol. 1, p. 1.

${ }^{11}$ O. A. Williams, A. Kriele, J. Hees, M. Wolfer, W. Müller, and C. E. Nebel, Chem. Phys. Lett. 495, 84 (2010).

${ }^{12}$ UNCD grown by Advanced Diamond Technologies.

${ }^{13}$ M. Imboden and P. Mohanty, Phys. Rev. B 79, 125424 (2009).

${ }^{14}$ S. Zaitsev, O. Shtempluck, E. Buks, and O. Gottlieb, Nonlinear Dyn. 67, 859 (2012).

${ }^{15}$ E. Collin, J. Kofler, S. Lakhloufi, S. Pairis, Y. Bunkov, and H. Godfrin, J. Appl. Phys. 107, 114905 (2010).

${ }^{16}$ J. Suh, M. LaHaye, P. Echternach, K. Schwab, and M. Roukes, Nano Lett. 10, 3990 (2010).

${ }^{17}$ J. Nichol, E. Hemesath, L. Lauhon, and R. Budakian, Appl. Phys. Lett. 95, 123116 (2009).

${ }^{18}$ R. Almog, S. Zaitsev, O. Shtempluck, and E. Buks, Phys. Rev. Lett. 98, 78103 (2007).

${ }^{19}$ A. Eichler, J. Moser, J. Chaste, M. Zdrojek, I. Wilson-Rae, and A. Bachtold, Nat. Nanotechnol. 6, 339 (2011).

${ }^{20}$ R. König, M. Ramos, I. Usherov-Marshak, J. Arcas-Guijarro, A. Hernando-Maneru, and P. Esquinazi, Phys. Rev. B 65, 180201 (2002). 